U289, Hopital de la Salpetriere, 47 boulevard de l'Hopital, 75651 Paris Cedex 13, France).

COMMENT. In this large series of patients with CMTX, differences between male and female patients are more apparent than in previous studies. Males are more severely affected than females, and onset before 10 years is also more frequent in males than females, occurring in $27 \%$ and $15 \%$ of patients, respectively. Patients with early onset present nonfunctional genetic mutations. These gender differences are also reflected in electrophysiological findings.

\title{
EEG IN LATE INFANTILE NEURONAL CEROID LIPOFUSCINOSIS
}

The clinical and electroencephalographic findings in 18 cases ( 8 girls, 10 boys) with late infantile neuronal ceroid lipofuscinosis (NCL) are reported from the University of Genova, and University of Siena, Italy. Age at onset ranged from 1 year 6 months to 6 years 6 months. Presenting symptoms were seizures in 12 patients, ataxia in 4 , and psychomotor regression followed by seizures in 2 . All patients had developed a progressive myoclonic epilepsy at follow-up. Neuroimaging in $12 / 18$ patients showed cerebral atrophy in 6 and cerebellar atrophy in 4 . Electron microscopy of skin, conjunctiva or rectal biopsies showed characteristic curvilinear bodies and fingerprint profiles.

EEG findings confirmed the classical and diagnostic pseudoperiodic atypical high voltage slow spike and wave pattern in $15 / 18$ cases, as previously reported, and some additional variants are described. While awake, background activity is slowed and pseudoperiodic discharges are at intervals of 8-10 sec. The response to eye opening and closing is absent, and the periodic pattern remains unmodified. During sleep, there is an absence of sleep spindles, and the periodic discharges are less evident. Treatment with ACTH partially controlled seizures but failed to block the periodic pattern. (Veneselli E, Biancheri R, Buoni S, Fois A. Clinical and EEG findings in 18 cases of late infantile neuronal ceroid lipofuscinosis. Brain Dev August 2001;23:306-311). (Respond: Dr Edvige Veneselli, Child Neuropsychiatry Unit, G Gaslini Institute, University of Genova, Largo G Gaslini 5, 16147 Genova, Italy).

COMMENT. The value of the EEG in the early diagnosis of late infantile neuronal ceroid lipofuscinosis is confirmed by this study of 18 cases. The first report of the characteristic pseudoperiodic pattern found in cases of LINCL was that of Pampiglioni G and Harden A in 1977, at Great Ormond Street Children's Hospital, London, UK. Subsequent reports of the EEG in LINCL and other types of NCL from the same center include those of Boyd SG and Harden A (1991), and Williams RE, Boyd SG, Lake BD, and Wilson J (1997). Recently, molecular genetic analyses have clarified the diagnosis in some NCL variants.

The classification of various forms of neuronal ceroid lipofuscinosis is described by Dyken PR (In Progress in Pediatric Neurology II. PNB Publishers, 1994;481-485). These include: Infantile (Santavuori), Late Infantile (Bielschowsky), Early Juvenile (Zeman-Dyken), Juvenile (Batten), Adult Recessive (Kufs), Adult Dominant (Boehme), and Atypical (Dyken-Wisniewski). Fifteen atypical forms of NCL are identified, and 21 subtypes, most representing separate diseases with different chromosome defects: chromosome 1 defect in the infantile form; 13 in early juvenile, 16 in the juvenile form, and neither chromosome 1,13 , nor 16 in the LINCL. (Dyken, 1994). 